\title{
Comparison of Multiple Sclerosis Cortical Lesion Types Detected by Multicontrast 3T and 7T MRI
}

\author{
(D). Maranzano, (D) M. Dadar, (DD.A. Rudko, (DD. De Nigris, (D) Elliott, DJ.S. Gati, DS.A. Morrow, DR.S. Menon, (DD.L. Collins,
} (DD.L. Arnold, and IDS. Narayanan

수을

\begin{abstract}
BACKGROUND AND PURPOSE: Our aims were the following: 1) to compare multicontrast cortical lesion detection using 3T and 7T MR imaging, 2) to compare cortical lesion type frequency in relapsing-remitting and secondary-progressive MS, and 3) to assess whether detectability is related to the magnetization transfer ratio, an imaging marker sensitive to myelin content.
\end{abstract}

MATERIALS AND METHODS: Multicontrast 3T and 7T MR images from 10 participants with relapsing-remitting MS and 10 with secondaryprogressive MS. We used the following 3T contrast sequences: 3D-T1-weighted, quantitative T1, FLAIR, magnetization-transfer, and 2D proton-density- and T2-weighted. We used the following 7T contrast sequences: 3D-T1-weighted, quantitative T1, and 2D-T2*-weighted.

RESULTS: Cortical lesion counts at 7T were the following: 720 total cortical lesions, 420 leukocortical lesions (58\%), 27 intracortical lesions (4\%), and 273 subpial lesions (38\%). Cortical lesion counts at 3T were the following: 424 total cortical, 393 leukocortical (93\%), zero intracortical, and 31 subpial (7\%) lesions. Total, intracortical, and subpial 3T lesion counts were significantly lower than the 7T counts $(P<.002)$. Leukocortical lesion counts were not significantly different between scanners. Total and leukocortical lesion counts were significantly higher in secondary-progressive MS, at 3T and 7T $(P \leq .02)$. Subpial lesions were significantly higher in secondary-progressive MS at 7T $(P=.006)$. The magnetization transfer ratio values of leukocortical lesions visible on both scanners were significantly lower than the magnetization transfer ratio values of leukocortical lesions visible only at $3 \mathrm{~T}$. No significant difference was found in magnetization transfer ratio values between subpial lesions visible only at 7T and subpial lesions visible on both $3 \mathrm{~T}$ and $7 \mathrm{~T}$.

CONCLUSIONS: Detection of leukocortical lesions at 3T is comparable with that at 7T MR imaging. Imaging at 3T is less sensitive to intracortical and subpial lesions. Leukocortical lesions not visible on 7T T2*-weighted MRI may be associated with less demyelination than those that are visible. Detectability of subpial lesions does not appear to be related to the degree of demyelination.

ABBREVIATIONS: $\mathrm{CL}$ = cortical lesion; $\mathrm{IC}=$ intracortical; $\mathrm{LC}=$ leukocortical; $\mathrm{MTR}=$ magnetization transfer ratio; $\mathrm{NAcGM}=$ normal-appearing cortical GM; RRMS = relapsing-remitting MS; SP = subpial; SPMS = secondary-progressive MS

ultiple sclerosis is an inflammatory and neurodegenerative disease characterized by lesions that affect the white mat-

\footnotetext{
Received February 3, 2019; accepted after revision May 10
}

From the Department of Anatomy (J.M.), University of Quebec in Trois-Rivieres, Trois-Rivieres, Quebec, Canada; McConnell Brain Imaging Centre (J.M., M.D., D.A.R. D.D.N., C.E., D.L.C., D.L.A., S.N.), Montreal Neurological Institute, McGill University, Montreal, Quebec, Canada; Department of Biomedical Engineering (M.D., D.A.R. D.L.C.), McGill University, Montreal, Quebec, Canada; Centre for Functional and Metabolic Mapping (J.S.G., R.S.M.), Robarts Research Institute, University of Western Ontario, London, Ontario, Canada; Department of Clinical Neurological Sciences (S.A.M.), London Health Sciences Centre, University of Western Ontario, London, Ontario, Canada; and Department of Medical Biophysics (R.S.M.), University of Western Ontario, London, Ontario, Canada

This study was supported by a Canadian Institutes of Health Research Grant (grant No. 201303MOP-302444- MPI-CFAA-23603)

This article is dedicated to the memory our dear friend and colleague, Dr Marcelo Kremenchutzky, Chair of Clinical MS Research at London Health Sciences Centre and a leading MS neurologist and clinician-scientist, who recently passed away. With regard to the current work, Dr Kremenchutzky made substantial contribu- ter and gray matter of the central nervous system. ${ }^{1}$ MR imaging of the brain is the criterion standard method to detect MS lesions in vivo, ${ }^{2}$ allowing an accurate quantification of the WM component of MS. However, correlations between a patient's clinical status and WM lesion load remain modest ${ }^{3,4}$, so it is

tions to the conception and design of this study and the acquisition and interpretation of data, and he critically revised the manuscript for intellectual content. May he rest in peace.

Please address correspondence to Josefina Maranzano, MD, PhD, University of Quebec in Trois-Rivieres, Department of Anatomy. Pavillon Leon-Provancher, Local 3501. 3351, Boulevard des Forges, Trois-Rivieres, QC, Canada. G8Z 4M3; McGill University, Department of Neurology and Neurosurgery, Montreal Neurological Institute, 3801 Rue University, Room WB327, Montreal, QC, Canada, H3A 2B4; e-mail: josefina.maranzano@uqtr.ca and josefina.maranzano@mail.mcgill.ca

-- Indicates open access to non-subscribers at www.ajnr.org

三 Indicates article with supplemental on-line tables.

Indicates article with supplemental on-line photo

http://dx.doi.org/10.3174/ajnr.A6099 
Table 1: Demographic and clinical characteristics of the participants

\begin{tabular}{|c|c|c|c|}
\hline & $\begin{array}{l}\text { Patients with } \\
\text { RRMS }\end{array}$ & $\begin{array}{l}\text { Patients with } \\
\text { SPMS }\end{array}$ & $\begin{array}{c}\text { Significant Differences } \\
\text { ( } P \text { Value) }\end{array}$ \\
\hline Male/female & $3: 7$ & $3: 7$ & \\
\hline \multirow[t]{2}{*}{ EDSS score } & Median, 1.5 & Median, 4.5 & $.001^{\mathrm{a}}$ \\
\hline & Range, 1-3 & Range, 3-6.5 & \\
\hline \multirow[t]{2}{*}{ Age at Onset (yr) } & Mean, 34 & Mean, 30 & $.15^{\mathrm{b}}$ \\
\hline & SD, 8.2 & SD, 6.5 & \\
\hline \multirow[t]{2}{*}{ Disease duration (yr) } & Mean, 12 & Mean, 18 & $.04^{\mathrm{b}}$ \\
\hline & SD, 7.8 & $\mathrm{SD}, 6.8$ & \\
\hline \multirow{2}{*}{$\begin{array}{l}\text { White matter lesion } \\
\text { volume }\left(\mathrm{cm}^{3}\right)\end{array}$} & Median, 9.3 & Median, 12.9 & $.18^{\mathrm{a}}$ \\
\hline & Range, $0.8-44.3$ & Range, 3.7-50.4 & \\
\hline
\end{tabular}

Note:-EDSS indicates Expanded Disability Status Scale.

${ }^{a}$ Mann-Whitney $U$ test

b $T$ test.

essential to accurately quantify GM lesions, especially in the cortex, which could increase clinical-imaging correlations.

MS cortical lesions (CLs) have been classified histopathologically into 4 types: type I leukocortical (LC), affecting the cortex and juxtacortical white WM; type II intracortical (IC), affecting only the cortex, without reaching the WM or subpial surfaces; type III subpial (SP), affecting the outer cortex along the SP boundary but not reaching the WM surface; and type IV, also SP but cortex-spanning, affecting all layers of the cortex. ${ }^{5}$ In many studies, types III and IV are grouped together as SP.

In vivo, CLs are detectable using MR imaging but are more difficult to visualize than WM lesions because of the lower myelin content in the cortex. Consequently, the change in myelin-related signal in CLs is smaller. CLs also generally exhibit less inflammation than WM lesions, ${ }^{6}$ leading to limited alteration of T1 and T2. ${ }^{4,7,8}$

During the past 2 decades, there has been significant interest in improving MR imaging-based detection of CLs. ${ }^{8-12}$ At MR imaging field strengths of $1.5 \mathrm{~T}$ and $3 \mathrm{~T}$, CLs are difficult to classify due to an insufficient signal-to-noise ratio (SNR), making the delineation of the boundaries between small CLs and surrounding normal tissue very challenging. ${ }^{10}$ At 7T, a classification of CLs that approximates that of histology is possible because of the increased SNR afforded by high-field MR imaging; hence, 7T MR imaging has emerged as the in vivo criterion standard for CL identification. ${ }^{8,13,14}$

The reduced sensitivity of lower MR imaging field strengths for CL detection is reflected in postmortem studies. In particular, 1.5T and 3T MR imaging detect only a small fraction of the CLs, as low as $5 \% .{ }^{15} \mathrm{MR}$ imaging at $7 \mathrm{~T}$ improves the detection rate ${ }^{16}$; however, a large number of CLs are still not captured. This is particularly true for type III lesions, for which 7\%-32\% detection sensitivities have been reported. ${ }^{14}$

The simultaneous use of multiple MR imaging contrasts improves CL detection over single-contrast reads at $3 \mathrm{~T}$, so multicontrast $3 \mathrm{~T}$ MR imaging CL quantification may provide results closer to those from $7 \mathrm{~T} .{ }^{17}$

Our study presents the assessment of CL counts obtained with multicontrast reading protocols on both $3 \mathrm{~T}^{17}$ and $7 \mathrm{~T} \mathrm{MR}$ images of the same MS cohort. Our goal was to establish which CL types were visible at $3 \mathrm{~T}$ compared with 7T, using a multicontrast approach on coregistered images and to assess whether detectability was related to the degree of abnormality on magnetization transfer imaging, ${ }^{18,19}$ as a measure of relative myelin content.

\section{MATERIALS AND METHODS Study Population}

Twenty participants with MS (6 men, 14 women, between 38 and 56 years of age) followed at the London, Ontario MS clinic, were recruited as part of a larger prospective cohort study designed to assess CLs in MS. The first 10 participants with relapsing-remitting MS (RRMS) and the first 10 with secondary-progressive MS (SPMS) were included in the present cross-sectional analysis. The median Expanded Disability Status Scale score was 3 (range, 1-6.5). The demographic details of the participants are presented in Table 1.

The study was approved by the institutional research ethics boards of the University of Western Ontario and McGill University. Each participant gave written informed consent.

\section{MR Imaging Acquisition}

All participants were imaged at the Centre for Functional and Metabolic Mapping at the Robarts Research Institute, University of Western Ontario. Subjects were imaged on a 3T Magnetom Prisma MR imaging scanner (Siemens, Erlangen, Germany). The same subjects were then scanned on a 7T Magnetom Step 2.3 (Siemens), using an 8-channel parallel-transmit configuration. Each participant was scanned at both $3 \mathrm{~T}$ and $7 \mathrm{~T}$ on the same day, except for 1 person whose scanning sessions were a week apart. None of the patients received treatment with corticosteroids during the month before the MR imaging.

The 3T MR imaging contrast sequences acquired were the following: 1) 3D-MP2RAGE sequence, yielding a 3D-T1-weighted image and a quantitative T1-map; 2) 3D-FLAIR; 3) 2D dual-echo, TSE, yielding proton-density-weighted and T2-weighted images; and 4) 3D-FLASH with and without a magnetization transfer pulse, to compute the magnetization transfer ratio (MTR) images.

On the 7T scanner, we obtained MP2RAGE and 2D-FLASH $\mathrm{T} 2{ }^{*}$-weighted image volumes. The detailed acquisition parameters are listed in Table 2.

\section{MR Imaging Analysis}

Image Processing. All 3T MR imaging contrast sequences were coregistered before CL segmentation, using the following imageprocessing pipeline: 1) brain mask extraction, ${ }^{20}$ 2) bias field correction, ${ }^{21,22} 3$ ) linear 9-parameter registration of the T1-weighted image to standard Montreal Neurological Institute stereotactic space $^{23}$ (transformations for registration were calculated using gradient orientations of minimal uncertainty), ${ }^{24} 4$ ) intermodality 6-parameter registration, and 5) resampling of all modalities to the T1-weighted image in Montreal Neurological Institute stereotactic space. $^{25}$

7T images were bias-field-corrected, ${ }^{21}$ and the MP2RAGE volumes (3D-T1WI and T1 map) were then linearly registered to the 
Table 2: MRI acquisition parameters

\begin{tabular}{|c|c|c|c|c|c|c|}
\hline & 3T TIWI & $\begin{array}{c}\text { 3T PDw/ } \\
\text { T2WI }\end{array}$ & $\begin{array}{c}3 T \\
\text { FLAIR }\end{array}$ & $\begin{array}{l}\text { 3T MT } \\
\text { On/Off }\end{array}$ & 7T TIWI & 7T T2* \\
\hline Sequence & $\begin{array}{c}\text { 3D- } \\
\text { MP2RAGE }\end{array}$ & $\begin{array}{l}\text { 2D-dual } \\
\text { echo TSE }\end{array}$ & 3D-TSE & 3D-GRE & 3D-MP2RAGE & 2D-GRE \\
\hline Orientation & Sagittal & Axial & Sagittal & Axial & Sagittal & Axial \\
\hline $\mathrm{TR}(\mathrm{ms})$ & 5000 & 2350 & 6000 & 36 & 6000 & 1000 \\
\hline TE (ms) & 2.98 & $\begin{array}{l}22 \\
87\end{array}$ & 356 & 3.86 & 2.7 & 22 \\
\hline TI (ms) & $\begin{array}{r}700 \\
2500\end{array}$ & NA & 2200 & NA & $\begin{array}{r}800 \\
2700\end{array}$ & NA \\
\hline Flip angle & $\begin{array}{l}4^{\circ} \\
5^{\circ}\end{array}$ & $120^{\circ}$ & $180^{\circ}$ & $10^{\circ}$ & $\begin{array}{l}4^{\circ} \\
5^{\circ}\end{array}$ & $55^{\circ}$ \\
\hline Slices & 176 & 120 & 176 & 192 & 224 & 60 (2 Stacks of 30) \\
\hline Voxel size (mm) & $1 \times 1 \times 1$ & $1 \times 1 \times 1.5$ & $1 \times 1 \times 1$ & $1 \times 1 \times 1$ & $0.7 \times 0.7 \times 0.7$ & $0.3 \times 0.3 \times 1$ \\
\hline Scan time (min:sec) & $8: 22$ & $5: 26$ & $8: 44$ & $8: 10$ & 10:14 & 8:26 (per stack) \\
\hline
\end{tabular}

Note:-PDw indicates proton density-weighted; MT, magnetization transfer; GRE, gradient recalled-echo; NA, not applicable.

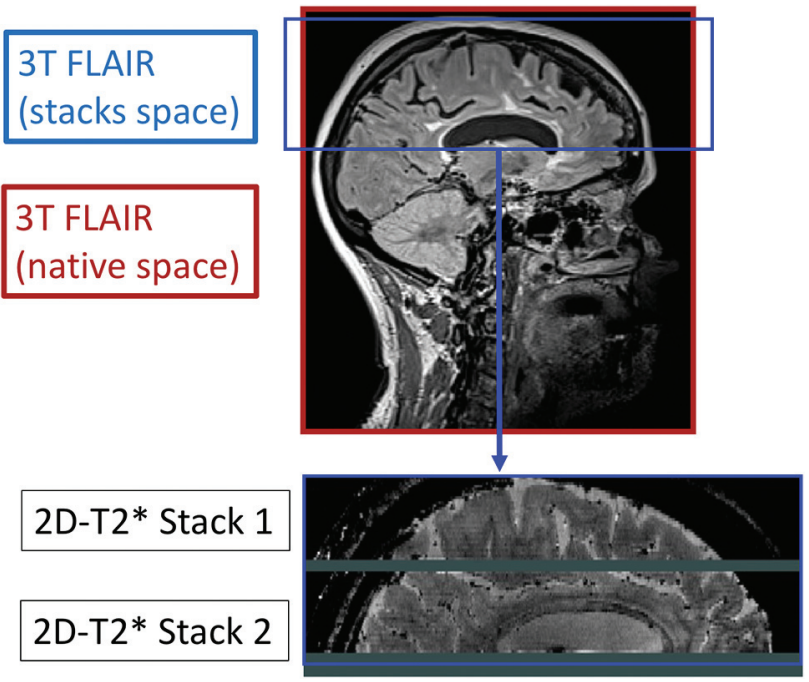

FIG 1. Multicontrast protocol volumetric area. Due to the sensitivity of $7 \mathrm{~T} \mathrm{T2} 2^{*}$-weighted acquisitions to magnetic susceptibility inhomogeneities present at the level of the posterior fossa and inferior temporal lobes, coverage of this interleaved, multislice acquisition is limited to the superior temporal lobes and above. Representation of the common volume of the brain is considered when comparing CL types detected on $3 \mathrm{~T}$ and 7T.

$\mathrm{T}^{*}$-weighted images, maintaining the high in-plane resolution of the $\mathrm{T} 2{ }^{*}$-weighted image that allows the classification of CL types. ${ }^{8}$

Once CL identification was performed (see the Cortical Lesion Quantification section below), we linearly registered all 3T MR imaging contrast sequences and their corresponding masks to 7T T2*WI space. This allowed accurate assessment of colocalization between CLs identified at 3T and 7T.

All comparisons between $3 \mathrm{~T}$ and $7 \mathrm{~T}$ scans were performed in the same volumetric area covered by the $7 \mathrm{~T} \mathrm{~T} 2{ }^{*} \mathrm{WI}$ because this sequence does not include the inferior portion of the temporal lobes or the posterior fossa, while the $3 \mathrm{~T}$ scans cover the whole brain (Fig 1).

Cortical Lesion Quantification. All MR images were segmented by an experienced rater (J.M. with $>13$ years of experience in lesion segmentation on MR imaging research scans of patients with MS), blinded to clinical status. Intra- and interrater reliability was assessed in 2 datasets (details presented in On-line Tables 1 and 2, respectively).
3T CL identification was performed in random order before 7T CL identification, with an interval of 3 months between reads.

CLs were manually segmented using the interactive software package "Display" (https://github.com/BIC-MNI/Display) developed at the McConnell Brain Imaging Center of the Montreal Neurological Institute. This program allows simultaneous viewing and lesion segmentation in the coronal, sagittal, and axial planes and cycling between multiple coregistered image contrasts. The program provides a "painting" tool for marking voxels with a given color (label number). These labels are saved in a separate label file that can be loaded for superimposition onto other brain images. ${ }^{26}$

On 3T images, CLs were segmented and classified in a single group, ${ }^{17}$ providing a total CL count. This was done because the resolution at $3 \mathrm{~T}$ does not allow a confident classification of CL types. Lesions had to be at least 3 voxels and have a high signal intensity on FLAIR and T2WI/proton-density weighted imaging and low signal intensity on T1WI in relation to the surrounding normal-appearing GM to be considered a 3T CL.

On 7T images, CLs were segmented as 3 different types (using 3 label numbers) following the classification by Bø et al $(2003)^{5,27}$ : type I (LC), type II (IC), and types III/IV (SP). Figure 2 shows an example of these different types of lesions on our 7T T2*WI. All 7T contrasts were used to segment 7T CLs, which had to be at least 3 voxels and be hyperintense on $\mathrm{T} 2^{\star} \mathrm{WI}$ and the T1 map and hypointense on the $3 \mathrm{D}$-T1WI contrast sequence in relation to the surrounding normal-appearing GM.

Once all scans were read, the 3T MR imaging contrast scans and CL masks of each patient were registered to the corresponding $7 \mathrm{~T} \mathrm{~T} 2{ }^{\star} \mathrm{WI}$ contrast. A side-by-side comparison of registered image slices allowed the categorization of CLs into those that had been identified on both 3T and 7T images (common lesions), CLs only identified at 3T (7T-negative lesions), and CLs only identified at 7T (3T-negative lesions). It also allowed us to determine the type of CL identified by $3 \mathrm{~T}$ images: retrospective classification of $3 \mathrm{~T}$ CL types.

White Matter Lesion Quantification. WM lesions were segmented using an automated Bayesian classifier followed by manual review and correction by an experienced rater (J.M.), as previously described. ${ }^{28}$ The contrasts used for the classification and 

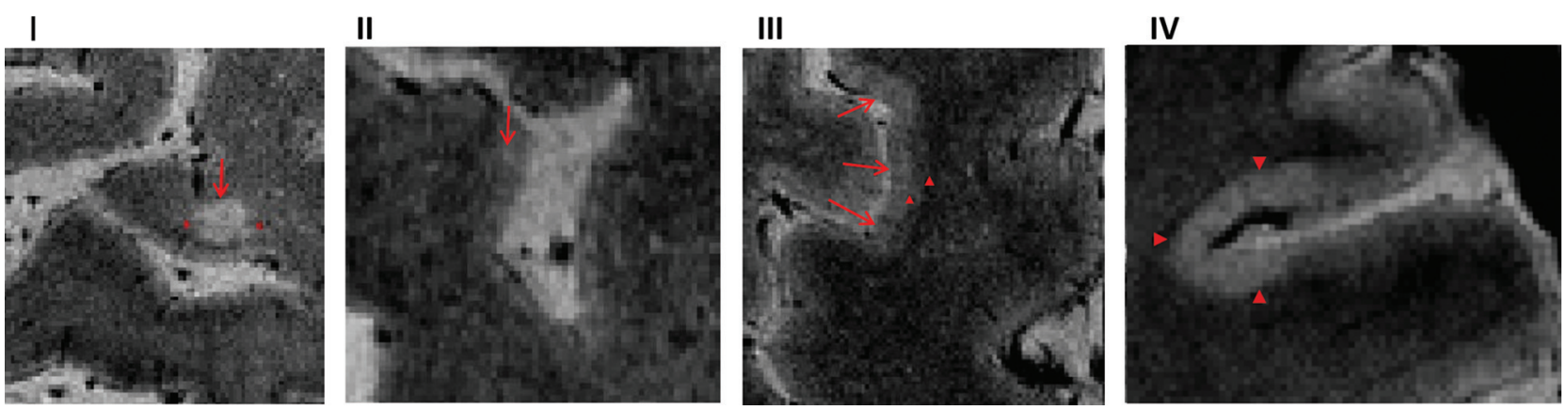

FIG 2. Cortical lesion classification. $7 \mathrm{~T} \mathrm{T2*} \mathrm{MR} \mathrm{imaging} \mathrm{cortical} \mathrm{lesion} \mathrm{classification} \mathrm{(Bø} \mathrm{et} \mathrm{al} \mathrm{2003).}{ }^{5}$

manual correction of the WM lesions were 3T T1WI, FLAIR, and $\mathrm{T} 2 \mathrm{WI} /$ proton-density-weighted.

Normal-Appearing Cortical Gray Matter Segmentation. Masks of normal-appearing cortical GM (NAcGM) adjacent to each CL were created by dilating the CL masks by 5 voxels in the 3 coordinate directions. A cortical GM tissue mask based on a multiatlas label fusion method ${ }^{29}$ was used to avoid the inclusion of juxtacortical WM and CSF.

\section{Statistical Analysis}

Subject demographic data that were normally distributed were compared using a $t$ test; otherwise, a Mann-Whitney $U$ test was used. CL counts (3T versus 7T) were compared using a Wilcoxon signed rank test. The frequency of CL types in RRMS and SPMS was compared using a $\chi^{2}$ test. The relationship between CL counts at $3 \mathrm{~T}$ and $7 \mathrm{~T}$ was evaluated with the Spearman correlation, and sensitivity [3T True-Positive/(3T True-Positive + 3T FalseNegative) $\times 100$ ], specificity [3T True-Negative/(3T True-Negative $+3 \mathrm{~T}$ False-Positive $) \times 100]$, positive predictive value $[3 \mathrm{~T}$ True-Positive/(3T True-Positive +3 T False-Positive $) \times 100]$, and negative predictive value $[3 \mathrm{~T}$ True-Negative/(3T TrueNegative $+3 \mathrm{~T}$ False-Negative $) \times 100]$ of $3 \mathrm{~T}$ counts with respect to $7 \mathrm{~T}$ counts were calculated.

An Generalized Linear Model with a negative binomial distribution and a log-link function assessed the relationship of CL count as the outcome variable to the disease type (RRMS versus SPMS) as the predictor factor, adjusting for disease duration and age at symptom onset:

$$
\begin{aligned}
\text { CL Count } \sim(\text { Log-Link Function }) \text { Disease Type } \\
\\
\quad+\text { Age at Symptom Onset }+ \text { Disease Duration. }
\end{aligned}
$$

The same type of GLM was used to determine the relationship between different CL types (LC and SP) and 4 predictor variables: 1) disease type, 2) age at symptom onset, 3) disease duration, and 4) WM lesion volume:

LC/SP Lesion Count $\sim($ Log-Link Function) Disease Type

$$
+ \text { Age at Symptom Onset + Disease Duration }
$$

$$
\text { + WM Lesion Volume. }
$$

We also assessed whether the number of SP lesions detected by $7 \mathrm{~T}$ MR imaging was related to the total number of CLs detected by 3T MR imaging, using the same type of GLM:
7T SP Lesion Count

(Log-Link Function) 3T Total CL count.

A negative binomial regression model was chosen in all cases in which the CL count was the outcome variable, due to its overdispersion. ${ }^{30}$

Finally, differences in MTR values across CL types were assessed and compared with the surrounding (NAcGM) using a mixed-effects model:

$$
\text { Mean MTR } \sim 1+\text { Lesion Type + (1| subject ID). }
$$

All statistical analyses were performed using MATLAB R2018a and SPSS v.24.

\section{RESULTS}

\section{Study Population Features}

The male-female ratio was the same in the RRMS and SPMS groups: $3 / 7$. The age at the onset of MS was not significantly different between RRMS and SPMS participants $(P=0.15)$. The disease duration was significantly longer for SPMS $(P=.04)$. The Expanded Disability Status Scale scores were significantly higher in the SPMS group $(P=.001)$ (Table 1$)$.

The volume of WM lesions was not significantly different between RRMS and SPMS groups $(P=0.18)$.

\section{Cortical Lesion Counts}

Seven hundred twenty CLs were detected using the 7T multicontrast protocol. The median CL count was 24.5 per participant (range, 0-119). Of these, 420 (58\%) were LC, 27 (4\%) were IC, and $273(38 \%)$ were SP.

Four hundred twenty-four CLs were detected using the $3 \mathrm{~T}$ multicontrast protocol (count limited to the region covered by the $7 \mathrm{~T} \mathrm{T2}{ }^{\star} \mathrm{WI}$ scan). The median CL count was 13 per participant (range, 1-59). Of these, 393 (93\%) were retrospectively determined to be LC, none were IC, and 31 (7\%) were SP. The total 3T CL count, in the complete $3 \mathrm{~T}$ volume, was 617 , with a median count of 24.5 per participant (range, 1-85).

The $3 \mathrm{~T}$ total CL, IC, and SP lesion counts were significantly lower than the respective $7 \mathrm{~T}$ counts $(P=0.39)$. The LC lesion count was not significantly different between $3 \mathrm{~T}$ and $7 \mathrm{~T}$.

One hundred twenty-six (30\%) LC lesions were detected only on 3T MR imaging and were missed on 7T. On retrospective review, these LC lesions were visible at 7T on the MP2RAGE sequence but were not labeled as lesions due to the low signal on $\mathrm{T} 2{ }^{\star} \mathrm{WI}$. These $126 \mathrm{LC}$ lesions did not show a preferential location 

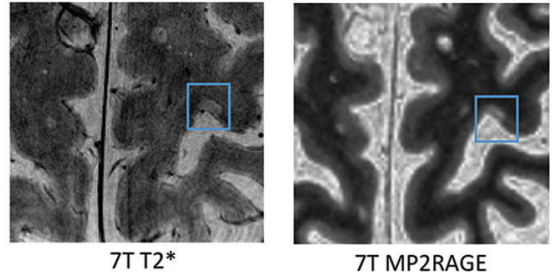

7T MP2RAGE

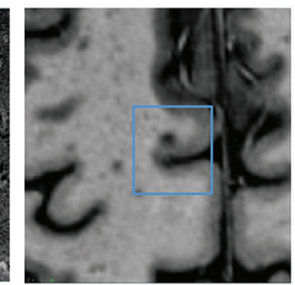

7T MP2RAGE

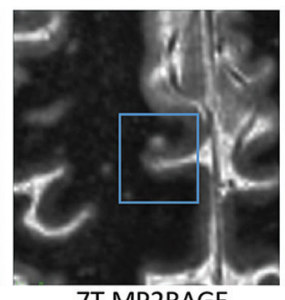

7T MP2RAGE

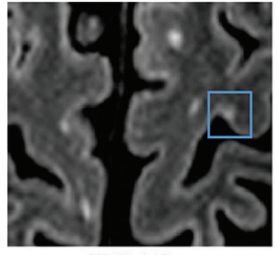

3T FLAIR

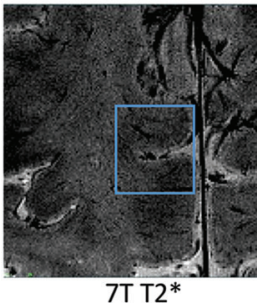

FIG 3. Example of $\mathrm{CL}$ lesions detected by $3 \mathrm{~T}$ and $7 \mathrm{~T}$ protocols. Example of $3 \mathrm{~T}-7 \mathrm{~T}$ common SP lesions (upper row) and 7T-negative LC lesions (lower row).

in the gyri; $43.6 \%$ affected the crown of the gyri, and $56.4 \%$ were located along the sulci. Finally, 50\% of these LC lesions were located in the frontal lobes (see detailed localization and lobe distribution in On-line Tables 3 and 4).

Thirty-one (11\%) SP lesions were detected on both 3T and 7T MR imaging (Fig 3).

At 7T total CL, LC, and SP lesion counts were significantly higher in SPMS participants after adjusting for disease duration and age at disease onset $(P=0.01, P=0.008$, and $P=0.03$, respectively). The IC lesion counts were not significantly different across MS types $(P=0.23)$, likely due to their small numbers ( 9 in RRMS, 18 in SPMS).

At $3 \mathrm{~T}$, the proportions of LC and SP lesions of the total CL count were not significantly different between RRMS and SPMS ( $P=0.06$ and $P=0.49$, respectively) (IC lesions were not detected at $3 \mathrm{~T}$ ). The proportions of CL types obtained on 7T MR imaging were significantly different between RRMS and SPMS when considering all $3 \mathrm{CL}$ types $(P=.02)$. However, when assessing differences between MS types in pairs of CL types, there were no significant differences in the proportions of LC and IC or IC and SP lesions. This finding is likely due to the small number of IC lesions that were identified. Conversely, the proportion of LC and SP lesions was significantly different between phenotypes $(P=0.007)$, with a higher proportion of LC lesions in SPMS. Details of the CL proportion by phenotype and tables used to calculate $\chi^{2}$ are presented in On-line Tables 5-9.

The correlation between CL counts obtained at 3T and 7T, determined using a nonparametric test (Spearman $\rho$ ), was 0.96 for LC lesions $(P<0.001)$ and 0.78 for SP lesions $(P<0.001)$ (Fig 4).

The sensitivity of $3 \mathrm{~T}$ counts with respect to $7 \mathrm{~T}$ counts was $67.2 \%$ for LC lesions and $11.3 \%$ for SP lesions. The specificity of $3 \mathrm{~T}$ counts, as well as the negative predictive values, could not be determined due to the inability to count true-negatives (cortex that appeared normal at both $3 \mathrm{~T}$ and $7 \mathrm{~T}$ ). The positive predictive values for the 3 T LC lesion count were $93.4 \%$ and $100 \%$ for SP lesions. However, these positive predictive values are relatively uninformative because the classification of CL 3T scans was only performed in a retrospective fashion.

\section{Cortical Lesion Magnetization Transfer Characteristics}

Mean MTR values of LC lesions visible on both scanners (3T-7T, common LC lesions) were significantly lower (mean MTR $=37.1 \pm 3.0)(P<0.001)$ than mean MTR values of LC lesions visible only on 3T (7T-negative LC lesions) (mean MTR $=38.3 \pm 2.6$ ).

The MTR of SP lesions visible on both 3T and 7T (common SP lesion) did not differ significantly from the MTR of SP lesions visible only on 7T (3T-negative SP lesion) $(P=.5)$.

The mean MTR values in the GM voxels of each lesion type were also compared with surrounding NAcGM MTR values. LC lesions only visible on $3 \mathrm{~T}$ scans or on both 3T and 7T scans showed significantly lower MTR values than the surrounding NAcGM $(P=.03$ and .002 , respectively). In LC lesions visible only on 7T scans, the MTR difference compared with the surrounding cortex did not reach statistical significance $(37.6 \pm 2.1$ versus $38.0 \pm 1.6, P=.06)$. IC lesions, only visible on $7 \mathrm{~T}$ scans, did not show lower MTR values than the surrounding NAcGM. Finally, SP lesions detected on either 7T or both 3T and 7T showed significantly lower MTR values compared with the surrounding NAcGM ( $P<0.001$ and .01 , respectively). Detailed MTR data are presented in On-line Table 10 and the On-line Figure.

\section{Association of Cortical Lesion Counts with Clinical and MR Imaging Measures}

We assessed the association between the 7T LC lesion count as the outcome variable and 4 predictors: MS type, disease duration, age at onset, and WM lesion volume. We found a significant association with SPMS $(P=.006)$, a significant negative association with age at onset $(P=.05)$, and a significant positive association with WM lesion volume $(P=.03)$. When the same associations were evaluated for SP lesion as the outcome variable, only SPMS was found to have a significant association $(P=.03)$.

Finally, we used a GLM to assess whether the total number of 3T CLs detected in the whole-brain volume could predict the number of SP lesions ( $3 \mathrm{~T}$ total CL count $=$ predictor; $7 \mathrm{~T} \mathrm{SP} \mathrm{lesion}$ count $=$ outcome variable), and we found a significant positive association $(P=.03)$.

\section{DISCUSSION}

Comparison of our 2 multicontrast MR imaging reading protocols, using coregistered images derived from 3T and 7T scans, showed that LC lesions are detected with a comparable degree of accuracy and acceptable sensitivity on 3T scans: 3T LC sensitivity $=67.2 \%$ and $3 \mathrm{~T}$ LC positive predictive value $=93.4 \%$. Furthermore, we found that the correlation between LC lesion counts on 3T and 7T was very high: $r=0.96, P<0.001$. Thus, a 3T multicontrast detection of LC lesions could be considered equivalent to a $7 \mathrm{~T}$ multicontrast assessment of LC lesions using $\mathrm{T}^{*}$ and MP2RAGE.

Our study also reproduces previous findings regarding the su- 

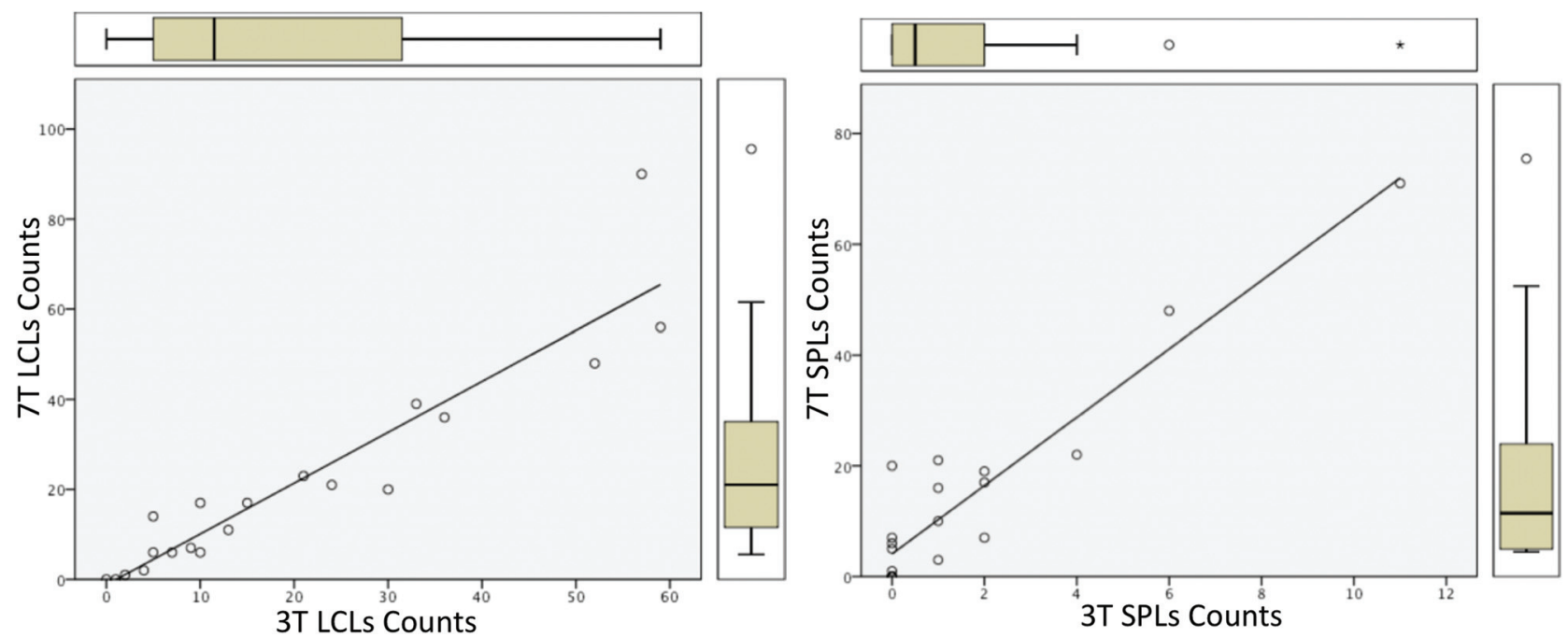

FIG 4. CL count correlations. Leukocortical and subpial lesion count correlations across scanners: $3 T$ LC lesion counts and 7T LC lesion counts; 3T SP lesion counts and 7T SP lesion counts.

perior sensitivity of 7T MR imaging in the detection of SP and IC lesions, with a $3 \mathrm{~T}$ sensitivity for SP lesions of only $11.3 \% .{ }^{14} \mathrm{~A}$ slice-by-slice comparison of $3 \mathrm{~T}$ images coregistered with $7 \mathrm{~T}$ images revealed that $11 \%$ of SP lesions detected on 3T MR imaging could not be prospectively differentiated from LC lesions due to the lower resolution of the $3 \mathrm{~T}$ scans. However, the number of 3T-positive SP lesions showed a high positive predictive value (100\%) and a high correlation with the total SP lesion count at 7T ( $r=0.78, P<0.001)$, suggesting that the few SP lesions detected on $3 \mathrm{~T}$ scans are all true-positives and that their distribution across participants would be representative of the distribution of SP lesions at 7T, albeit with a much lower sensitivity.

We also performed a slice-by-slice comparison of coregistered 3T and 7T images to assess CLs that were identified exclusively on $3 \mathrm{~T}$ or $7 \mathrm{~T}$ images. We found that $30 \%$ of LC lesions were not identified at $7 \mathrm{~T}$ due to the absence of increased signal on the $7 \mathrm{~T}$ T2 ${ }^{*} \mathrm{WI}$ contrast, though they exhibited clear hypointensity on 3D-T1WI at 7T and elevated signal on the T1 map. ${ }^{14}$ Conversely, these negative-7T LC lesions were visible on 3T FLAIR, ${ }^{13}$ where they exhibited the classic hyperintensity of MS lesions. The finding of LC lesions negative on 7T T2* WI is in line with previous studies that reported a lower sensitivity of $7 \mathrm{~T} \mathrm{T2*WI} \mathrm{compared}$ with 7T T2WI in the detection of LC lesions. ${ }^{14,31,32}$ Specifically, Kilsdonk et al, in 2016, ${ }^{14}$ performed an ex vivo comparison between 7T MR imaging CL detection by lesion type on various MR imaging contrast sequences versus histopathology, reporting that $7 \mathrm{~T} \mathrm{~T} 2{ }^{\star} \mathrm{WI}$ was able to detect only $50 \%$ of all LC lesions versus a detection of $100 \%$ using $7 \mathrm{~T}$ T2WI. Due to both time constraints and inherent challenges related to whole-head $\mathrm{B}_{1}$ shimming for T2WI acquisitions at 7T, it is not always possible to acquire both $\mathrm{T} 2{ }^{\star} \mathrm{WI}$ and $\mathrm{T} 2 \mathrm{WI}$ data in a single scan session. Consequently, researchers may favor $7 \mathrm{~T} \mathrm{T2}{ }^{\star} \mathrm{WI}$, given its more sensitive performance in the assessment of SP lesions, which is the most difficult CL type to detect. However, if an MP2RAGE sequence is used at $7 \mathrm{~T}$, the sensitivity for LC lesions might be improved by increasing the weight of joint positivity on 3D-T1WI and the T1 map and relaxing the requirement for hyperintensity on T2*WI. Alternatively, the inclusion of a FLAIR contrast in the acquisition proto- col of 7T scans may increase the LC lesion detection rate when combined with the MP2RAGE sequence.

Our finding that 7T-negative LC lesion had significantly higher MTR values than those found in 3T-7T common LC lesion suggests that negative LC lesion on $7 \mathrm{~T}$ might be related to lower levels of demyelination. ${ }^{19}$ Additionally when comparing MTR values of LC lesions with the values of the surrounding NAcGM, 3T-negative LC lesions showed lower MTR values but did not reach statistical significance $(P=.06)$. Conversely, common LC lesions and 7T negative did show significantly lower MTR values than the surrounding NAcGM $(P=0.002$ and $P=0.03$, respectively).

MTR was also used to characterize SP lesions visible on both 3T and 7T (common SP lesions) versus SP lesions only visible on 7T (3T-negative SP lesions). We did not find significant differences in MTR values, suggesting that the visibility of SP lesions on 3T images is not related to their degree of myelination/demyelination, ${ }^{19}$ but possibly to MR imaging resolution and T2 contrast characteristics of the lesions. We speculate that the inherently lower myelin content in cortical GM, particularly in the superficial layers, ${ }^{33}$ may also, in part, be responsible for the lack of MTR differences between common SP lesions and 3T-negative SP lesions. However, when we performed the comparison of MTR values of these lesions with the surrounding NAcGM, both common SP lesions and 3T-negative SP lesions showed significantly lower values $(P=0.01$ and $P<0.001$, respectively).

Our study also assessed differences in CL types in the RRMS and SPMS groups. We did not find a higher proportion of SP lesions in SPMS, as suggested by ex vivo studies. ${ }^{34,35}$ This discrepancy could be related to the use of end-stage SPMS tissue samples in previous ex vivo studies. The participants with SPMS in our study had moderate disability and were of similar age to the those with RRMS. Another possible explanation could be related to pathologists typically only considering regions of complete demyelination as true lesions. ${ }^{35}$ This type of definition is operationally impossible on MR imaging in vivo. When one applies MR imaging for in vivo identification of CLs, some LC lesions could represent areas of partial demyelination. ${ }^{32}$ This would increase the 
MR imaging-based LC count in relation to a corresponding count using histology. In addition, on MR imaging, we were able to assess lesion counts over a larger volumetric area of the brain, as opposed to single slices evaluated in ex vivo studies.

Regarding the associations of CL counts and clinical and MR imaging measurements as predictors, we observed a significant association with MS type of both LC and SP lesions, with significantly higher counts in SPMS. However, while LC lesion counts also showed a significant positive association with WM lesion volumes and a significant negative association with age at disease onset, SP lesions did not. These differences could be related to variations in the dynamic aspects of the pathophysiologic evolution in the 2 lesion types. However, given the lower sensitivity of MR imaging to SP lesions, our sample size may be too small to observe significant associations with other predictors. ${ }^{14}$

Finally, the positive relationship of 3T total CL count to the 7T SP count, along with the strong relationship between LC lesion count at $3 \mathrm{~T}$ and $7 \mathrm{~T}$, suggests that the overall CL identification performed on $3 \mathrm{~T}$ is informative regarding the overall CL burden. The use of multicontrast acquisitions and reading protocol would allow many more centers without access to 7T scanning to assess CL pathology in MS.

\section{Limitations}

The absence of a histopathologic assessment is an obvious limitation of this study. As a consequence, we have characterized lesions as $3 \mathrm{~T}$ positive/negative and 7T positive/negative because previous work using ex vivo MR imaging has shown that 7T still has sensitivity limitations in the detection of the different CL types. ${ }^{14} \mathrm{Nev}-$ ertheless, we performed an analysis of sensitivity and calculated predictive values of $3 \mathrm{~T}$ counts in relation to $7 \mathrm{~T}$ counts, given the generally higher performance of 7T imaging. An ex vivo study has shown that partial demyelination in CLs is detectable on MR imaging $^{32}$; however, most ex vivo studies do not consider partially demyelinated lesions in their quantifications. We believe that this is a limiting factor in the interpretation of count data in comparing MR imaging and histology results.

\section{CONCLUSIONS}

3T multicontrast MR imaging is an adequate method to detect the number of LC lesions. 7T MR imaging is necessary for quantifying SP lesions and IC lesions in vivo. However, CLs detected at 3T include a fraction of SP pathology with a high predictive value that significantly correlates with SP lesions seen on 7T. This outcome suggests that a standardized, multicontrast 3T MR imaging reading protocol on coregistered images can be a valuable method to assess overall CL burden in vivo. We found that LC and SP lesions were higher in participants with SPMS, highlighting their association with progression in MS. Longitudinal studies are needed to assess the rate of accumulation of CLs and their association with change in clinical variables.

\section{ACKNOWLEDGMENTS}

We thank Dr Erin Beck, Clinical Fellow at the Translational Neuroradiology Section, Division of Neuroimmunology and Neurovirology, National Institute of Neurologic Disorders and Stroke, National Institutes of Health, who performed 7T cortical lesion segmentations in the dataset used to calculate the interrater reliability results.

Disclosures: Josefina Maranzano-RELATED: Grant: Canadian Institutes of Health Research*; UNRELATED: Grants/Grants Pending: Multiple Sclerosis Society of Canada, Comments: doctoral award from July 1, 2018, up to November 2018 (I completed my PhD in November, so the award was closed). . Colm Elliott-UNRELATED: Consultancy: F. Hoffmann-La Roche Ltd, Comments: Participated on an advisory board as a representative of NeuroRx Research*; Employment: NeuroRx Research. Sarah A. Morrow-RELATED: Grant: Canadian Institutes of Health Research.* Ravi S. Menon-RELATED: Grant: Canadian Institutes for Health Research.* D. Louis Collins-UNRELATED: Consultancy: NeuroRx Research, Comments: consultancy and training on image-processing procedures; Employment: McGill University, Comments: I am a professor at McGill University. Douglas L. Arnold-RELATED: Grant: Canadian Institutes of Health Research*; UNRELATED: Consultancy: Acorda Therapeutics, Biogen, Celgene, GeNeuro, Genentech, Hoffmann-La Roche, Merck, Novartis, Roche, Sanofi, Teva Pharmaceutical Industries, and Wave Life Sciences; Employment: NeuroRx Research; Grants/Grants Pending: Canadian Institutes of Health Research, Multiple Sclerosis Society of Canada, Biogen, Novartis, Immunotec; Stock/ Stock Options: NeuroRx Research. Sridar Narayanan-UNRELATED: Employment: NeuroRx Research, Comments: Part-time; Grant: Immunotec. *Money paid to the Institution.

\section{REFERENCES}

1. Compston A, Coles A. Multiple sclerosis. Lancet 2008;372:1502-17 CrossRef Medline

2. Reich DS, Lucchinetti CF, Calabresi PA. Multiple sclerosis. $N$ Engl J Med 2018;378:169-80 CrossRef Medline

3. Rocca MA, Messina R, Filippi M. Multiple sclerosis imaging: recent advances. J Neurol 2013;260:929-35 CrossRef Medline

4. Calabrese M, Filippi M, Gallo P. Cortical lesions in multiple sclerosis. Nat Rev Neurol 2010;6:438-44 CrossRef Medline

5. Bø L, Vedeler CA, Nyland HI, et al. Subpial demyelination in the cerebral cortex of multiple sclerosis patients. J Neuropathol Exp Neurol 2003;62:723-32 CrossRef Medline

6. Peterson JW, Bö L, Mörk S, et al. Transected neurites, apoptotic neurons, and reduced inflammation in cortical multiple sclerosis lesions. Ann Neurol 2001;50:389-400 CrossRef Medline

7. Absinta M, Sati P, Reich DS. Advanced MRI and staging of multiple sclerosis lesions. Nat Rev Neurol 2016;12:358-68 CrossRef Medline

8. Mainero $\mathrm{C}$, Benner $\mathrm{T}$, Radding $\mathrm{A}$, et al. In vivo imaging of cortical pathology in multiple sclerosis using ultra-high field MRI. Neurology 2009;73:941-48 CrossRef Medline

9. Seewann A, Kooi EJ, Roosendaal SD, et al. Postmortem verification of MS cortical lesion detection with 3D DIR. Neurology 2012;78: 302-08 CrossRef Medline

10. Geurts JJ, Roosendaal SD, Calabrese M, et al; MAGNIMS Study Group. Consensus recommendations for MS cortical lesion scoring using double inversion recovery MRI. Neurology 2011;76:418-24 CrossRef Medline

11. Sethi V, Yousry TA, Muhlert N, et al. Improved detection of cortical MS lesions with phase-sensitive inversion recovery MRI. J Neurol Neurosurg Psychiatry 2012;83:877-82 CrossRef Medline

12. Nelson F, Poonawalla A, Hou P, et al. 3D MPRAGE improves classification of cortical lesions in multiple sclerosis. Mult Scler 2008;14: 1214-19 CrossRef Medline

13. Kilsdonk ID, de Graaf WL, Soriano AL, et al. Multicontrast MR imaging at 7T in multiple sclerosis: highest lesion detection in cortical gray matter with 3D-FLAIR. AJNR Am J Neuroradiol 2013;34:791-96 CrossRef Medline

14. Kilsdonk ID, Jonkman LE, Klaver R, et al. Increased cortical grey matter lesion detection in multiple sclerosis with 7 T MRI: a postmortem verification study. Brain 2016;139:1472-81 CrossRef Medline

15. Geurts JJ, Bö L, Pouwels PJ, et al. Cortical lesions in multiple sclerosis: combined postmortem MR imaging and histopathology. AJNR Am J Neuroradiol 2005;26:572-77 Medline

16. Nielsen AS, Kinkel RP, Tinelli E, et al. Focal cortical lesion detection 
in multiple sclerosis: 3 Tesla DIR versus 7 Tesla FLASH-T2. J Magn Reson Imaging 2012;35:537-42 CrossRef Medline

17. Maranzano J, Rudko DA, Arnold DL, et al. Manual segmentation of MS cortical lesions using MRI: a comparison of 3 MRI reading protocols. AJNR Am J Neuroradiol 2016;37:1623-28 CrossRef Medline

18. Brown RA, Narayanan S, Arnold DL. Segmentation of magnetization transfer ratio lesions for longitudinal analysis of demyelination and remyelination in multiple sclerosis. Neuroimage 2013;66: 103-09 CrossRef Medline

19. Chen JT, Easley K, Schneider C, et al. Clinically feasible MTR is sensitive to cortical demyelination in MS. Neurology 2013;80:246-52 CrossRef Medline

20. Eskildsen SF, Coupé P, Fonov V, et al; Alzheimer's Disease Neuroimaging Initiative. BEaST: brain extraction based on nonlocal segmentation technique. Neuroimage 2012;59:2362-73 CrossRef Medline

21. Sled JG, Zijdenbos AP, Evans AC. A nonparametric method for automatic correction of intensity nonuniformity in MRI data. IEEE Trans Med Imaging 1998;17:87-97 CrossRef Medline

22. Tustison NJ, Avants BB, Cook PA, et al. N4ITK: improved N3 bias correction. IEEE Trans Med Imaging 2010;29:1310-20 CrossRef Medline

23. Dadar M, Fonov VS, Collins DL. A comparison of publicly available linear MRI stereotaxic registration techniques. Neuroimage 2018; 174:191-200 CrossRef Medline

24. De Nigris D, Collins DL, Arbel T. Multi-modal image registration based on gradient orientations of minimal uncertainty. IEEE Trans Med Imaging 2012;31:2343-54 CrossRef Medline

25. Maranzano J, Till C, Assemlal HE, et al; Canadian Pediatric Demyelinating Disease Network. Detection and clinical correlation of leukocortical lesions in pediatric-onset multiple sclerosis on multi-contrast MRI. Mult Scler 2018 May 1. [Epub ahead of print] CrossRef Medline
26. Maranzano J, Rudko DA, Nakamura K, et al. MRI evidence of acute inflammation in leukocortical lesions of patients with early multiple sclerosis. Neurology 2017;89:714-21 CrossRef Medline

27. Bø L, Vedeler CA, Nyland H, et al. Intracortical multiple sclerosis lesions are not associated with increased lymphocyte infiltration. Mult Scler 2003;9:323-31 CrossRef Medline

28. Elliott C. A Bayesian Framework for 4-D Segmentation of Multiple Sclerosis Lesions in Serial MRI in the Brain. Doctoral thesis. Montreal: McGill University; 2016

29. Sabuncu MR, Yeo BT, Van Leemput K, et al. A generative model for image segmentation based on label fusion. IEEE Trans Med Imaging 2010;29:1714-29 CrossRef Medline

30. Sormani M, Stromillo ML, Battaglini M, et al. Modelling the distribution of cortical lesions in multiple sclerosis. Mult Scler 2012;18: 229-31 CrossRef Medline

31. Jonkman LE, Klaver R, Fleysher L, et al. Ultra-high-field MRI visualization of cortical multiple sclerosis lesions with $\mathrm{T} 2$ and $\mathrm{T} 2{ }^{\star}$ : a postmortem MRI and histopathology study. AJNR Am J Neuroradiol 2015;36:2062-67 CrossRef Medline

32. Yao B, Hametner S, van Gelderen P, et al. 7 Tesla magnetic resonance imaging to detect cortical pathology in multiple sclerosis. PLoS One 2014;9:e108863 CrossRef Medline

33. Palomero-Gallagher N, Zilles K. Cortical layers: cyto-, myelo-, receptor- and synaptic architecture in human cortical areas. Neuroimage 2017 Aug 12. [Epub ahead of print] CrossRef Medline

34. Kutzelnigg A, Lucchinetti CF, Stadelmann C, et al. Cortical demyelination and diffuse white matter injury in multiple sclerosis. Brain 2005;128:2705-12 CrossRef Medline

35. Kutzelnigg A, Lassmann H. Pathology of multiple sclerosis and related inflammatory demyelinating diseases. Handb Clin Neurol 2014;122:15-58 CrossRef Medline 